\title{
Two-Vector FCS-MPC for Permanent-Magnet Synchronous Motors Based on Duty Ratio Optimization
}

\author{
Long Sheng, ${ }^{1}$ Dapeng Li ${ }^{D},{ }^{2}$ and Yue $\mathrm{Ji}^{3}$ \\ ${ }^{1}$ College of Engineering, China University of Petroleum-Beijing at Karamay, Karamay 834000, China \\ ${ }^{2}$ College of Automation Engineering, Nanjing University of Aeronautics and Astronautics, Nanjing 211106, China \\ ${ }^{3}$ Consumer Customer Support Wuxi Center, Microsoft Corp., Block A, Dolphin, Wuxi Software Park, \\ No. 111 Linghu Ave., Wuxi 214135, China \\ Correspondence should be addressed to Dapeng Li; lidp401@163.com
}

Received 12 November 2017; Revised 27 March 2018; Accepted 30 April 2018; Published 31 May 2018

Academic Editor: Agathoklis Giaralis

Copyright (C) 2018 Long Sheng et al. This is an open access article distributed under the Creative Commons Attribution License, which permits unrestricted use, distribution, and reproduction in any medium, provided the original work is properly cited.

\begin{abstract}
The servo system of a permanent-magnet synchronous motor usually consists of current, speed, and position loops. Compared with conventional PI control, finite-control-set model predictive control (FCS-MPC) has the advantage of fast response. Conventional FCS-MPC relies on the precise parameters of system model and has large current ripple. To address that problem, this paper proposed an improved FCS-MPC based on duty ratio optimization in synchronous rotating reference frame. To get more precise voltage vector, the proposed FCS-MPC selects the optimal vector combination and, respectively, calculates the time duration. Moreover, feedback correction is also applied to improve the robustness of the control strategy. The simulation results validate the effectiveness of the algorithm.
\end{abstract}

\section{Introduction}

Thanks to their advantages of high efficiency, high power density, high reliability, and easy maintenance, permanentmagnet synchronous motors (PMSMs) have been widely used in some fields of high performance servo, including industrial robots, numerical control machine, and aerospace. In some occasions with high performance requirements, fast torque response is often required to ensure the high dynamic performance of the whole system. Presently, the control structure of PMSM servo system often contains 3 loops, which are current, speed, and position, respectively $[1,2]$. The 3 -loop control structure has the advantages of clear physical meaning and easy settings of parameters. However, the performance of current controller, in the inner loop, becomes a critical factor that influences the whole control performance.

Predictive control is a kind of computer control algorithm which develops from engineering. It has been implemented successfully in industrial process control and enjoys universal applicability. Being different from other control methods, predictive control evaluates the influence of control variables on the system and has faster dynamic performance compared with PI control. Deadbeat predictive control, generalized predictive control (GPC), predictive function control (PFC), and finite-control-set model predictive control (FCS-MPC) are all common predictive control methods $[3,4]$. Specifically, GPC consists of predictive model, rolling optimization, and feedback correction [5]. Unlike GPC, the control variable of $\mathrm{PFC}$ is a function constructed on process characteristics and tracking setting value [6]. Both of them are good at dealing with control constraints and multiobjective optimization, but massive online calculation limits PFC's wide application in real time occasions. Compared with GPC and PFC, the deadbeat predictive control has a simpler structure, since it leaves out the rolling optimization and feedback correction. It predicts the feedback value by taking the reference value and actual value into the model of controlled plant. But this control method is an open loop control. References [710] have proposed different methods to improve the steadystate performance of deadbeat predictive control, but those methods have limited effect on some motors with strong nonlinearities.

FCS-MPC is a new algorithm that combines the advantages of model predictive control, heuristic intellectual 


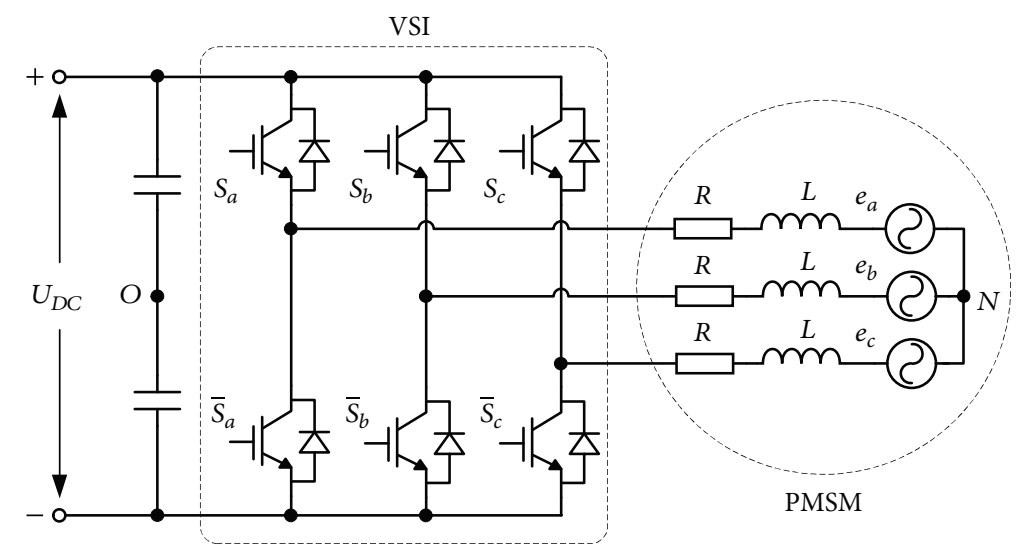

FIGURE 1: Simplified model of PMSM drive system.

optimization, and power electronic modulation. It can control current and torque of a converter [11-14]. Conventional FCS-MPC predicts current with 7 different voltage vectors and selects the most optimized vector with a cost function. It has faster response but larger current ripple [15]. A two-vector FCS-MPC based on direct torque control, which calculates the time duration of vectors, respectively, was put forward to reduce the current ripple [16]. However, this method ignores that the calculated time duration may exceed the whole control period and that uncertainties of model can cause poor steady-state performance.

This paper proposed an improved two-vector FCS-MPC based on duty ratio optimization. It combines with vector control and calculates the duty ratio of two voltage vectors on the prerequisite of minimizing the cost function. Moreover, the duty ratio is less than 1 . Meanwhile, a feedback correction is added to improve the robustness of the control strategy. Simulation results show that the proposed method has better steady-state performance and stronger robustness of current loop compared with the conventional one.

\section{Model Of PMSM}

The simplified model of surface-mounted PMSM driven by voltage source inverter in this paper is illustrated in Figure 1 and its mathematical model in synchronous rotating frame is expressed as

$$
\begin{aligned}
& u_{d}=R_{s} i_{d}+L_{d} \frac{d i_{d}}{d t}-\omega_{e} L_{q} i_{q} \\
& u_{q}=R_{s} i_{q}+L_{q} \frac{d i_{q}}{d t}+\omega_{e}\left(L_{d} i_{d}+\psi_{f}\right)
\end{aligned}
$$

where $u_{d}, u_{q}$ represent the voltages of direct and quadrature axes, respectively, $i_{d}, i_{q}$ represent the currents, $L_{d}, L_{q}$ are the inductances, $R_{s}$ is the resistance, $\omega_{e}$ is the rotor speed, and $\psi_{f}$ is the stator flux. For the surface-mounted PMSM, we could assume $L_{d} \approx L_{q}=L_{s}$.

When the sampling time $T_{s}$ is sufficiently small, we adopt the Euler approximation for the stator current derivatives [11],

$$
\frac{d i}{d t} \approx \frac{i(k+1)-i(k)}{T_{s}}
$$

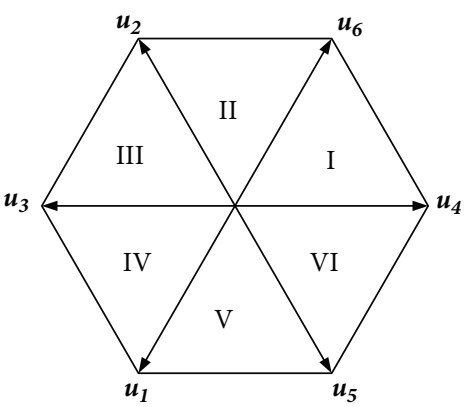

FIGURE 2: Voltage vectors and corresponding switching states.

Therefore, (1) can be discretized as

$$
\begin{aligned}
u_{d}(k)= & R_{s} i_{d}(k)+\frac{L_{d}}{T_{s}}\left[i_{d}(k+1)-i_{d}(k)\right] \\
& -\omega_{e}(k) L_{d^{2}} i_{q}(k) \\
u_{q}(k)= & R_{s} i_{q}(k)+\frac{L_{q}}{T_{s}}\left[i_{q}(k+1)-i_{q}(k)\right] \\
& +\omega_{e}(k)\left[L_{q} i_{d}(k)+\Psi_{f}\right]
\end{aligned}
$$

\section{Conventional Finite-Control-Set Model Predictive Control}

3-phase voltage source inverter has 8 different switching states, which correspond to 8 basic voltage vectors. Among them, there are 6 nonzero vectors and 2 zero vectors. As Figure 2 shows, the amplitude of the 6 active vectors is $2 / 3 E$ ( $E$ is the dc bus voltage) and the vector space is divided into 6 sections [7].

The discrete-time predictive model of PMSM can be written as follows:

$$
\begin{aligned}
i_{d}^{p}(k+1)= & \left(1-\frac{R_{0} T_{s}}{L_{d 0}}\right) i_{d}(k)+T_{s} \omega_{e} i_{q}(k)+\frac{T_{s}}{L_{d 0}} \\
& \cdot u_{d} \\
i_{q}^{p}(k+1)= & \left(1-\frac{R_{0} T_{s}}{L_{q 0}}\right) i_{q}(k)-T_{s} \omega_{e} i_{d}(k)-\psi_{f} \omega_{e} T_{s} \\
& +\frac{T_{s}}{L_{q 0}} \cdot u_{q}
\end{aligned}
$$




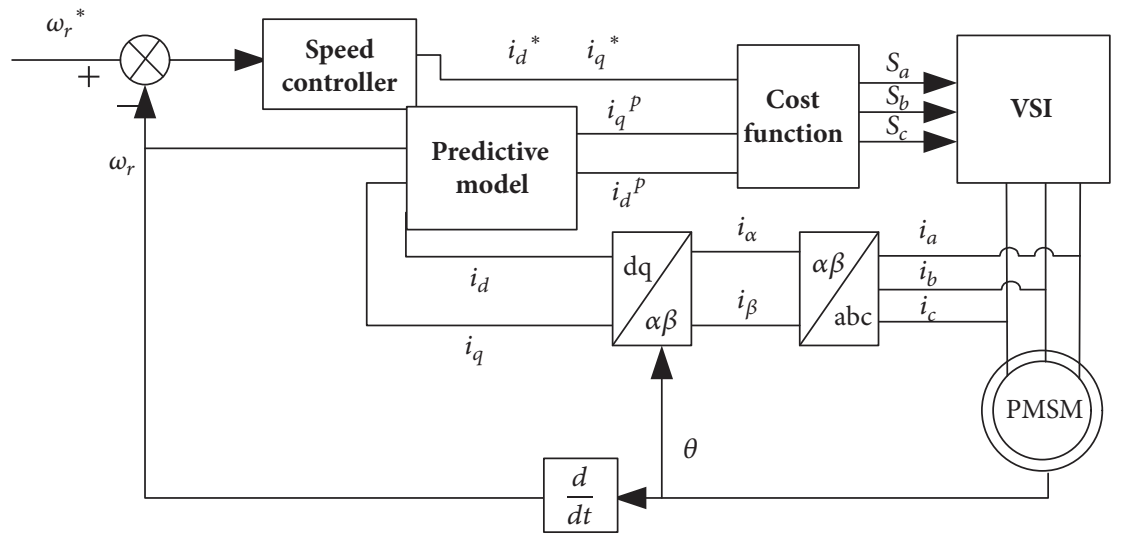

FIGURE 3: Control structure of model predictive control.

where $i_{d}{ }^{p}(k+1)$ and $i_{q}{ }^{p}(k+1)$ are the predictive current at $(k+1)$ th instant, $i_{d}(k), i_{q}(k)$ are the actual values of current at $k$ th instant, and $R_{0}, L_{d 0}$, and $L_{q 0}$ are the nominal values of resistance and inductance.

Figure 3 shows the control scheme of conventional FCSMPC, where the stator current components are predicted by the discrete-time predictive model. The voltage vector that minimizes the cost function is selected out from the 8 voltage vectors generated by the inverter and applied during a whole sampling interval [11].

Current control aims to minimize the error between reference current and actual current while keeping stator current below the maximum amplitude of current. These objectives can be expressed as the following cost function $[11,12]$ :

$$
\begin{aligned}
g= & {\left[i_{d}{ }^{p}(k+1)\right]^{2}+\left[i_{q}{ }^{*}(k)-i_{q}{ }^{p}(k+1)\right]^{2} } \\
& +f\left(i_{d}{ }^{p}(k+1), i_{q}{ }^{p}(k+1)\right)
\end{aligned}
$$

where the first item denotes the minimization of the reactive power, the second item is for precision tracking of the torqueproducing current, and the last item is a nonlinear function for limiting the amplitude of the stator current. This function can be expressed as [12]

$$
f\left(i_{d}{ }^{p}, i_{q}{ }^{p}\right)= \begin{cases}0 & \left|i_{d}{ }^{p}\right| \leq i_{d \max } \text { and }\left|i_{q}{ }^{p}\right| \leq i_{q \max } \\ \infty & \left|i_{d}{ }^{p}\right|>i_{d \max } \text { or }\left|i_{q}{ }^{p}\right|>i_{q \max }\end{cases}
$$

where $i_{d \max }$ and $i_{q \max }$ are the maxima allowed for the amplitudes of $d, q$ axis currents, respectively. On the one hand, if the predictive stator currents are below the limits, the cost function comprises only the first two items and the voltage vector that minimizes the current error will be selected. On the other hand, if a given voltage vector generates predictive currents with magnitudes higher than $i_{d \max }$ or $i_{q \max }$, the cost function will be infinitely great, and in turn the voltage vector will not be selected.

Given that the change of the high frequency switching states of an inverter will harm the steady-state performance of system, another constraint is imposed on the cost function to reduce the number of commutations of the power switches and minimize the switching losses. Therefore, the cost function should include an item that covers the number of switches that change [11] when the switching state $S(k)$ is applied, with respect to the previously applied switching state $S(k-1)$; that is,

$$
\begin{aligned}
g= & {\left[i_{d}{ }^{p}(k+1)\right]^{2}+\left[i_{q}{ }^{*}(k)-i_{q}{ }^{p}(k+1)\right]^{2} } \\
& +f\left(i_{d}{ }^{p}(k+1), i_{q}{ }^{p}(k+1)\right)+\lambda n
\end{aligned}
$$

where $\lambda$ is the weighting factor and $n$ is the number of switches that change when switching state $S(k)$ is applied. The switching state vector $S$ is defined as

$$
S=\left(S_{1}, S_{2}, S_{3}\right)
$$

where $S_{1}, S_{2}, S_{3}$ represent the states of a switch and only have two values, one or zero; then the number of switches that change from $S(k-1)$ to $S(k)$ is

$$
\begin{aligned}
n= & \sum_{x=1}^{N}\left|S_{x}(k)-S_{x}(k-1)\right| \\
= & \left|S_{1}(k)-S_{1}(k-1)\right|+\left|S_{2}(k)-S_{2}(k-1)\right| \\
& +\left|S_{3}(k)-S_{3}(k-1)\right|
\end{aligned}
$$

The influence of different values of the weighting factor $\lambda$ on the switching frequency is shown in Figure 4.

It can be seen that current ripple reduces when $\lambda$ improves, but the result is still not desirable for engineering, because only one voltage vector is applied in each control period, and when the predictive currents of the 8 vectors are unable to minimize the cost function, steady-state error appears.

\section{Two-Vector FCS-MPC Based on Duty Ratio Optimization}

In order to solve the problem caused by inaccurate voltage vector selection of conventional FCS-MPC, this paper proposes a two-vector FCS-MPC based on duty ratio optimization, which is an improvement to the conventional one. The 


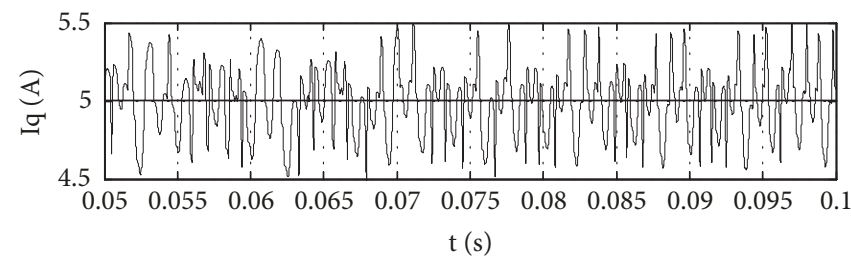

(a)

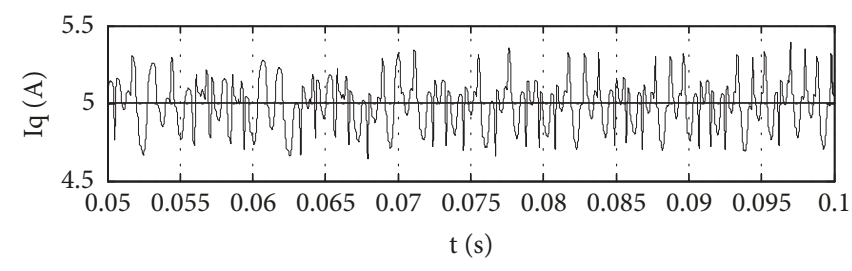

(b)

Figure 4: Influence of different $\lambda$ on current wave for (a) $\lambda=0.5$ and (b) $\lambda=0.8$.

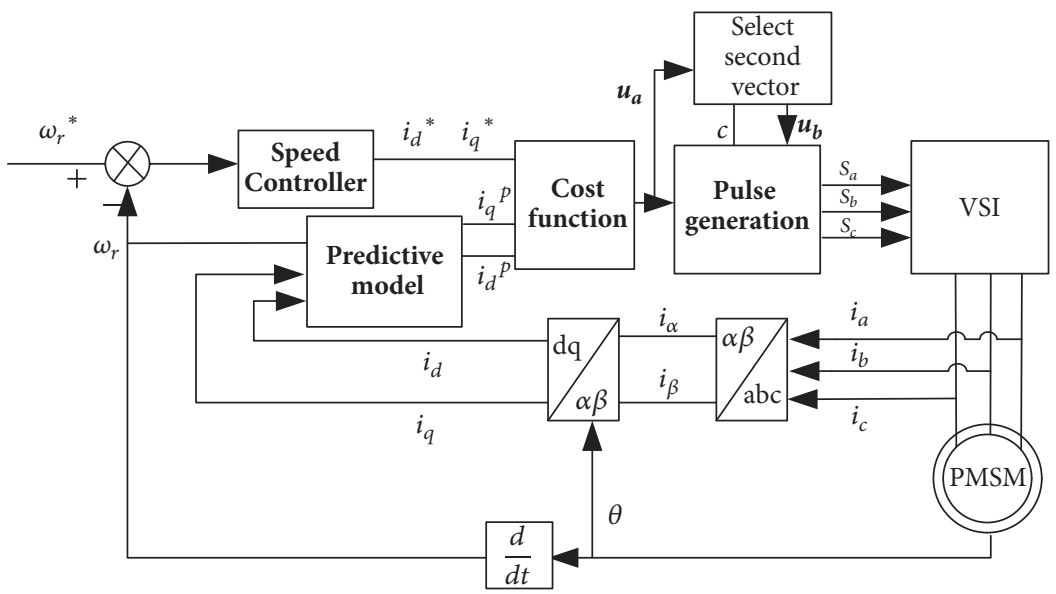

FIGURE 5: Control structure of two-vector FCS-MPC with duty ratio optimization.

proposed method uses the combination of two voltage vectors as the control set instead of only one vector [17]. In each control period, switching pulses can be generated according to the two vectors after calculating the duty ratio. The control scheme is shown in Figure 5.

Three situations of vector selection are considered, including two zero vectors, two nonzero vectors, one zero vector, and one nonzero vector. When the two vectors selected are the same, the control effect is the same as that of conventional FCS-MPC.

Similar to conventional FCS-MPC, the first active vector $\mathbf{u}_{\mathrm{a}}$ is selected by minimizing the cost function (7). To reduce complexity and avoid high switching frequency, the switching state should change only once in each control period. Hence, the second vector will be among adjacent vectors to $\mathbf{u}_{\mathrm{a}}$ or zero vector. For example, if $\mathbf{u}_{\mathbf{a}}$ is selected as $\mathbf{u}_{\mathbf{1}}(100)$, the second vector should be selected among $\mathbf{u}_{2}(110), \mathbf{u}_{6}(101)$, and $\mathbf{u}_{0}(000)$. For a determined vector $\mathbf{u}_{\mathbf{a}}$, there will be always three candidates of the second vectors, which means that $6 \times 3=18$ combinations are calculated [16] in the proposed method. The second vector's selection and duty ratio's calculation will be elaborated as follows. It is an effective method to directly compute the time duration of each vector in the condition of minimizing the cost function [17]. But with this method, the calculated time may exceed the whole control period, so the proposed control strategy uses the cost function to optimize duty ratio.

Assumed that $\mathbf{u}_{\mathbf{a}}$ is the first optimized vector and we get its duration time as follows:

$$
t_{a}=c * T_{s}
$$

where $t_{a}$ is the duration time of $\mathbf{u}_{\mathbf{a}}, c$ is the duty ratio in one control period. If the second vector is $\mathbf{u}_{\mathbf{b}}$, the applied voltage vector will be

$$
\mathbf{u}_{\mathbf{s}}=c T_{s} \mathbf{u}_{\mathbf{a}}+(1-c) T_{s} \mathbf{u}_{\mathbf{b}}
$$

Taking the quadrature axis as an example, the voltage equation of PMSM is

$$
\begin{aligned}
u_{q}(k)= & R_{0} \mathbf{i}_{q}+L_{q 0} \frac{i_{q}(k+1)-i_{q}(k)}{d t}+L_{q 0} \omega_{e} i_{d}(k) \\
& +T \psi_{f} \omega_{e}
\end{aligned}
$$

Here,

$$
u_{q}=c T_{s} u_{a q}+(1-c) T_{s} u_{b q}
$$

where $u_{a q}$ and $u_{b q}$ represent the voltages converted by inverse Park transformation of $q$ axis.

Considering (11), minimizing cost function (7) is equivalent to solving the following function:

$$
\frac{d(g)}{d c}=0
$$

From (11) to (14), the optimal duty ratio can be derived as

$$
\begin{aligned}
c= & \frac{\text { iqerr } \times\left(u_{a q}-u_{b q}\right)-i d e r r \times\left(u_{a d}-u_{b d}\right)}{\left(u_{a d}-u_{b d}\right)^{2}+\left(u_{a q}-u_{b q}\right)^{2}} \\
& -\frac{\left(T_{s}^{2} / L_{d 0}\right)\left(u_{a d}-u_{b d}\right) u_{2 d}+\left(T_{s}^{2} / L_{q 0}\right)\left(u_{a q}-u_{b q}\right) u_{b q}}{\left(u_{a d}-u_{b d}\right)^{2}+\left(u_{a q}-u_{b q}\right)^{2}}
\end{aligned}
$$




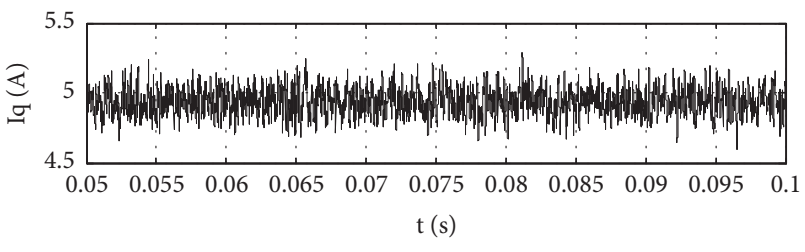

FIGURE 6: Steady-state current wave of two-vector FCS-MPC.

where $u_{a d}$ and $u_{b d}$ are the voltage converted by inverse Park transformation of $d$ axis, iqerr $=i_{q}{ }^{*}-\left(1-R_{0} T_{s} / L_{q 0}\right) \cdot i_{q}$, and iderr $=\left(1-R_{0} T_{s} / L_{d 0}\right) \cdot i_{d}+T_{s} \omega i_{q}$.

After two vectors and duty ratio are determined, to ensure the least switching transitions, the control sequence of voltage vector in the present control period should take last control period into consideration: for example, if the last vector applied during the last control period is $\mathbf{u}_{0}(000)$, and the vectors to be applied in present control period are $\mathbf{u}_{2}(110)$ and $\mathbf{u}_{3}(010)$. In this case, $\mathbf{u}_{3}(010)$ will be applied first instead of $\mathbf{u}_{2}(110)$. The steady-state current wave of two-vector FCSMPC is shown in Figure 6.

Therefore, there are two points in two-vector FCS-MPC. One is to select two optimal vectors and the other is to calculate the duty ratio. The objective of them is to minimize the cost function.

4.1. Feedback Correction. According to the classic theory of model predictive control, feedback correction should be added to make the control system a closed loop. Therefore, the proposed two-vector FCS-MPC is further improved by feedback correction [14].

Assume the current error of $q$ axis at $(k+1)$ th instant is $e$

$$
e(k+1)=i_{q}^{p}(k+1)-i_{q}(k+1)
$$

where $i_{q}{ }^{p}(k+1)$ is the predictive value, and $e(k+1)$ should be approximately equal to $e(k)$ because the value at $(k+1)$ th instant cannot be obtained.

$$
e(k)=i_{q}^{p}(k)-i_{q}(k)
$$

where $i_{q}{ }^{p}(k)$ is the predictive current of the last instant. If the sampling time is sufficiently small, the error could be considered as a constant in the prediction period; then the compensated predicted current $i_{q}{ }^{c}$ can be expressed as

$$
i_{q}^{c}(k+1)=i_{q}^{p}(k+1)+h e(k)
$$

where $h$ is the coefficient of feedback. Finally, the predictive current with feedback compensation will be applied into the cost function to select the most optimized voltage vector combination.

Still, taking the quadrature axis as an example, when the cost function reaches a minimum value, it will satisfy the necessary condition (14). Then $i_{q}{ }^{p}(k+1)$ can be derived by combining (18) with (14),

$$
i_{q}^{p}(k+1)=\frac{\left[i_{q}{ }^{*}(k)+h i_{q}(k)\right] z}{z+h}
$$

By taking (19) into the predictive model, the transfer function between reference current and actual current can be obtained as

$$
\begin{aligned}
& \frac{i_{q}(z)}{i_{q}{ }^{*}(z)} \\
& =\frac{\left(L_{q 0} / L_{q}\right) z^{2}}{z^{2}+\left[L_{q 0} / L_{q}+\left(1-L_{q 0} / L_{q}\right) h-1\right] z+\left(L_{q 0} / L_{q}-1\right) h}
\end{aligned}
$$

When $h=0$, the transfer function becomes

$$
\frac{i_{q}(z)}{i_{q}^{*}(z)}=\frac{\left(L_{q 0} / L_{q}\right) z}{z+\left(L_{q 0} / L_{q}\right)-1}
$$

According to Jury stability criterion, the stability condition can be derived as

$$
0<\frac{L_{q 0}}{L_{q}}<\frac{2-2 h}{1-2 h}
$$

And Figure 7 shows the root locus of the poles as $L_{q 0} / L_{q}$ varies.

It can be seen from Figure 7 that, for a specified $h$, the system is at a critical stable point when the inductance variation exceeds the range of (22). Meanwhile, smaller $h$ allows a higher degree of inaccuracy of inductance which improves the robustness of the system. It should be also noted that all the poles and the zeros cancel when $L_{q 0} / L_{q}=1$, and thus, an ideal model predictive control is obtained.

The control structure of the closed current loop with feedback correction is shown in Figure 8.

\section{Simulation Study}

In this section, the proposed two-vector FCS-MPC based on duty ratio optimization is simulated in the environment of MATLAB/Simulink. The performances of conventional FCSMPC, two-vector FCS-MPC, and two-vector FCS-MPC with feedback correction will be compared in detail. To verify the validity of the proposed method, the PI parameters of speed loop adopted in each control strategy are the same. Motor parameters in simulation are rated power $400 \mathrm{~W}$, rated speed $3000 \mathrm{rpm}$, stator resistance $2.35 \Omega$, inductance $L_{d}=L_{q}=$ $0.007 \mathrm{H}$, poles 4 , and inertial $6.5 \times 10^{-3} \mathrm{~kg} \cdot \mathrm{m}^{2}$.

A comparative study of conventional FCS-MPC, twovector FCS-MPC, and PI control is carried out in Figure 9, where the reference current is a step signal and the load is 4 N.m. The PI controller could be written as $P I(\mathrm{z})=K_{p}+$ $K_{i} /(z-1)$, where $K_{p}$ and $K_{i}$ are PI parameters. Besides, the feedforward compensation is also introduced in the PI control to eliminate the coupling of the $d q$ axes, and the control law could be written as

$$
\begin{aligned}
u_{d}(z)= & \underbrace{P I(z)\left[i_{d}^{*}(z)-i_{d}(z)\right]}_{u_{d 0}}-\underbrace{L_{q 0} \omega_{e}(z) i_{q}(z)}_{\text {Feedforward }}, \\
u_{q}(z)= & \underbrace{P I(z)\left[i_{q}^{*}(z)-i_{q}(z)\right]}_{u_{q 0}} \\
& +\underbrace{L_{d 0} \omega_{e}(z) i_{q}(z)+\psi_{f} \omega_{e}(z)}_{\text {Feedforward }} .
\end{aligned}
$$




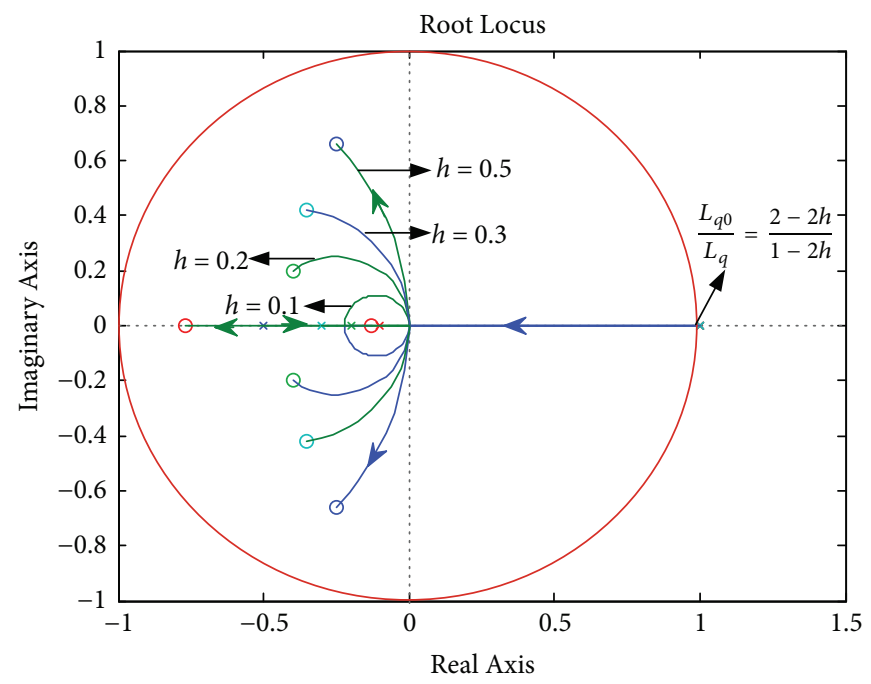

FIgURE 7: Root locus of poles with variation of $L_{q 0} / L_{q}$.

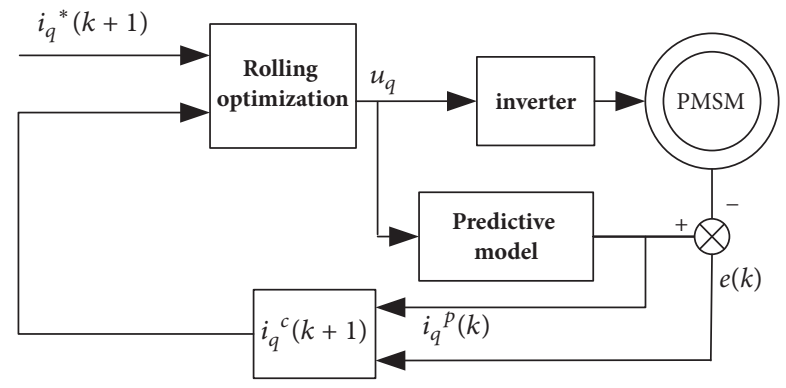

FIGURE 8: Control structure of FCS-MPC with feedback correction.

With the feedforward compensation based PI control, the PMSM current can be written as

$$
\begin{aligned}
& i_{d}(z)=\frac{K_{p}\left(z-1+K_{i} / K_{p}\right)}{z-1} \frac{T_{s}}{L_{d}\left(z-1+R_{s} T_{s} / L_{d}\right)}, \\
& i_{q}(z)=\frac{K_{p}\left(z-1+K_{i} / K_{p}\right)}{z-1} \frac{T_{s}}{L_{q}\left(z-1+R_{s} T_{s} / L_{q}\right)} .
\end{aligned}
$$

It can be seen that the PI controller could cancel the pole of PMSM when $K_{i} / K_{p}=R_{s} T_{s} / \mathrm{L}_{d 0 / q 0}$. Thus, in the simulation, we firstly set $K_{p}=24$ and $K_{i}=0.8$ to cancel the pole, and the closed-loop bandwidth is about $550 \mathrm{~Hz}$. However, those parameters would lead to overshoot and oscillation. Then we have tuned PI parameters by trial-and-error around the calculated values, and best value of the PI controller is $K_{p}=15$ and $K_{i}=0.8$, which are adopted in the simulation.

We can see that the current ripple is definitely larger than that of two-vector FCS-MPC based on duty ratio optimization, while the response of conventional FCS-MPC is slower. Besides, the PI controller is also slower than the proposed scheme. Furthermore, the 3-phase current wave shows that the wave of the proposed FCS-MPC gets closer to a sine wave that is needed in a PMSM. Also, their torque performances are compared in Figure 10, where the machine runs at $1000 \mathrm{rpm}$ and a step torque from $1 \mathrm{~N} . \mathrm{m}$ to 5 N.m is applied. The results reveal that conventional FCSMPC is unable to achieve satisfactory control performance when the load suddenly changes at $0.2 \mathrm{~s}$ due to the reason mentioned in Section 2, but the proposed FCS-MPC has better performance.

Additionally, the proposed FCS-MPC with feedback correction has better steady-state performance and robustness. The comparative results are illustrated in Figures 11 and 12 , where the nominal inductance is twice as much as the actual inductance and the machine runs at $3000 \mathrm{rpm}$ and starts without load. They indicate the validity of feedback correction. It can be seen from Figure 11 that the steady-state error decreases as $h$ increases. And when $h$ increases to a value, overshoot and delay will appear. Therefore, $h$ should be determined by adjusting.

\section{Conclusion}

Due to the application of only one voltage vector during one control period in conventional FCS-MPC, there are always large current ripple and poor steady-state performance. To tackle this problem, the present paper proposed a twovector FCS-MPC based on duty ratio optimization and the duty ratio is below 1 , which also solves the problem of invalid time duration calculated in the existing algorithm. Moreover, the uncertainties of model parameters will also influence control performance of FCS-MPC, so the proposed method introduced a feedback correction to improve the robustness. Simulation is carried out to verify the effectiveness of the proposed method. The results show that the proposed method exhibits smaller current ripple and better steady-state performance and is easy to implement. 

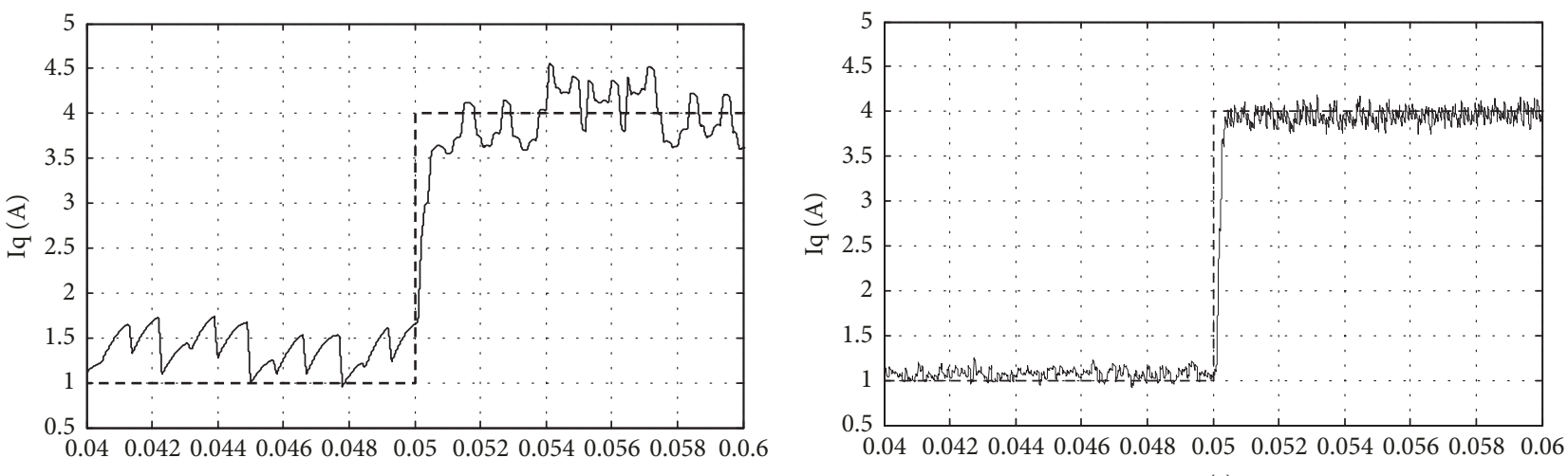

$\mathrm{t}(\mathrm{s})$
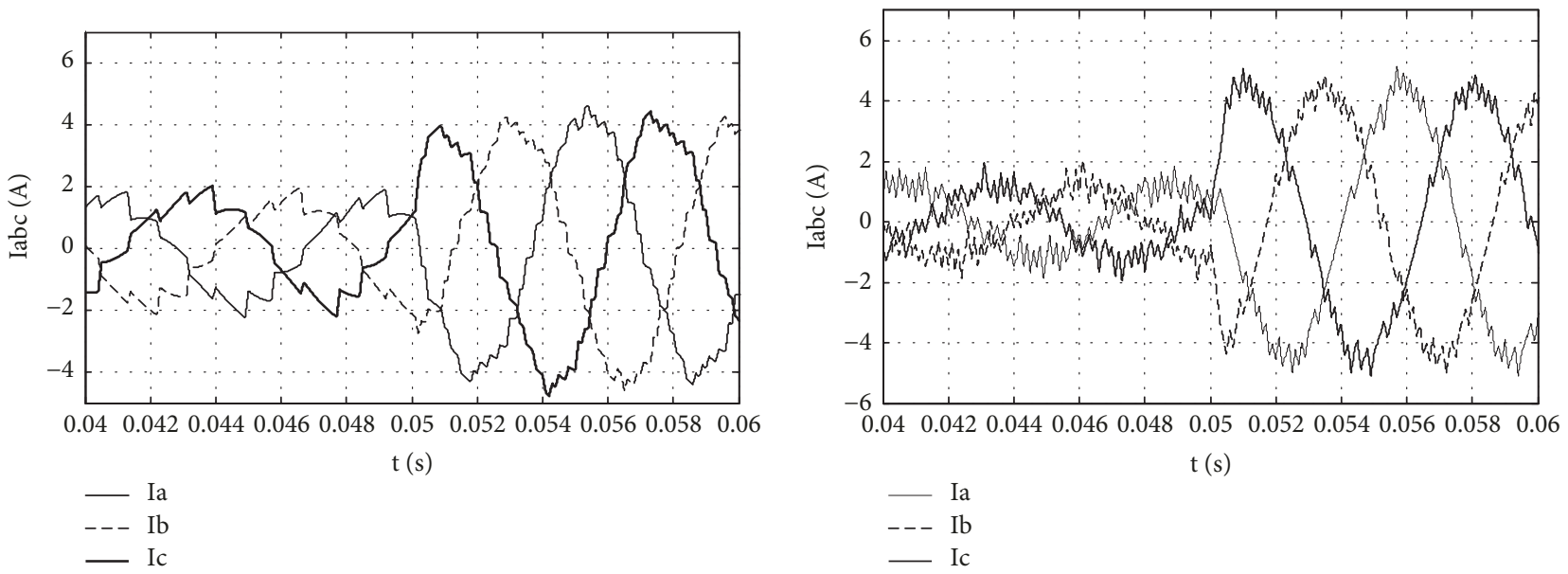

(a)

(b)
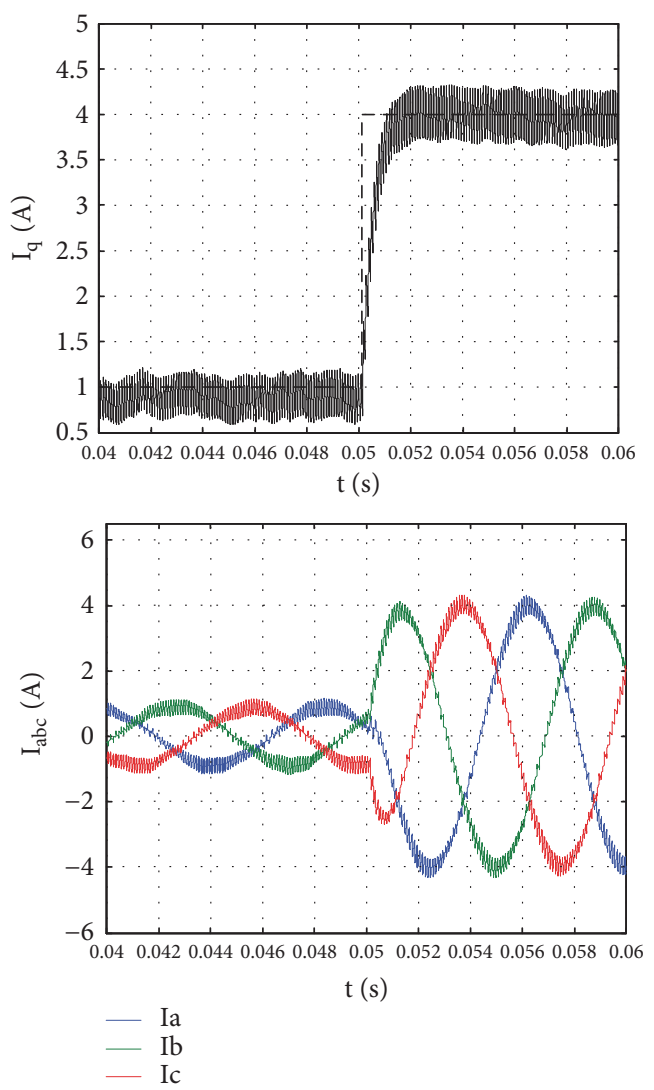

(c)

Figure 9: Simulated results of quadrature axis and 3-phase currents with sudden current change at $0.5 \mathrm{~s}$ and 4 N.m torque load for (a) conventional FCS-MPC, (b) two-vector FCS-MPC based on duty ratio optimization, and (c) PI control. 


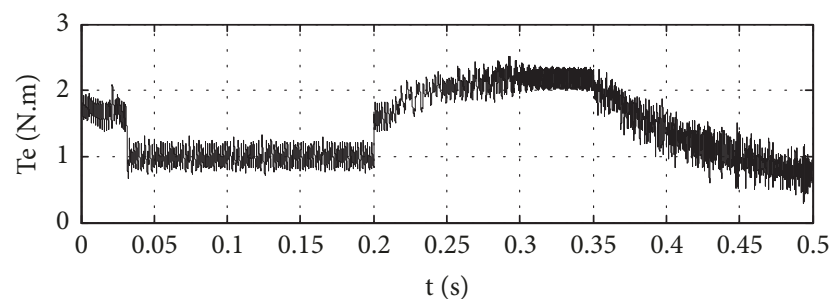

(a)

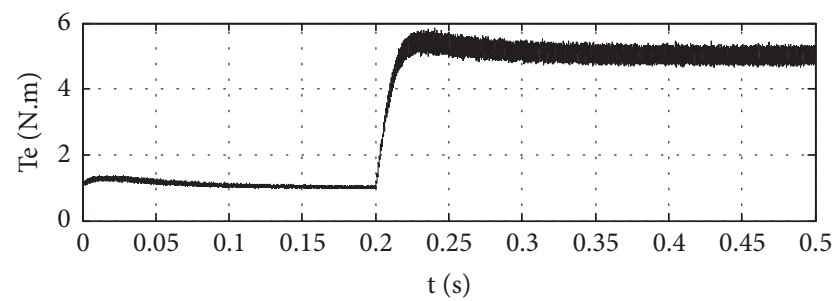

(b)

FIGURE 10: Simulated results of torque at $1000 \mathrm{rpm}$ with sudden load change at $0.2 \mathrm{~s}$ for (a) conventional FCS-MPC and (b) two-vector FCS-MPC based on duty ratio optimization.

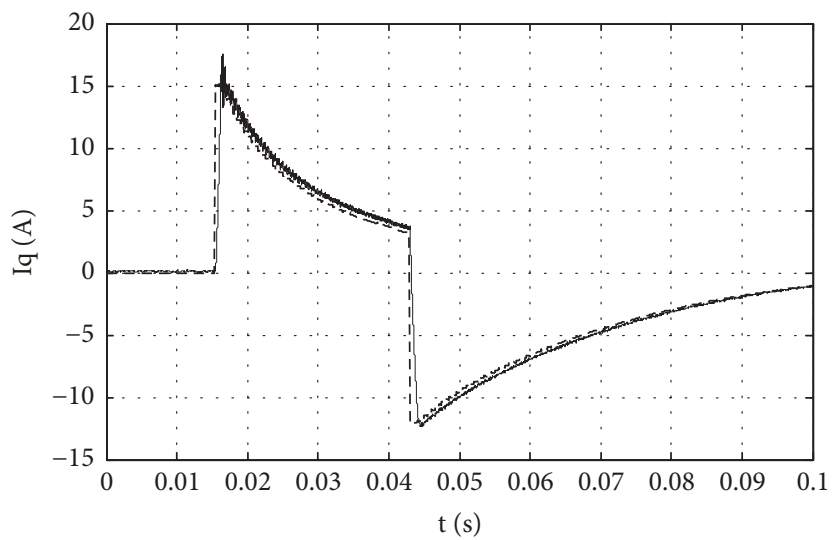

FIGURE 11: Simulated results of quadrature axis current with sudden speed change at $0.02 \mathrm{~s}$ and $0.06 \mathrm{~s}$ for two-vector FCS-MPC based on duty ratio optimization without feedback correction.

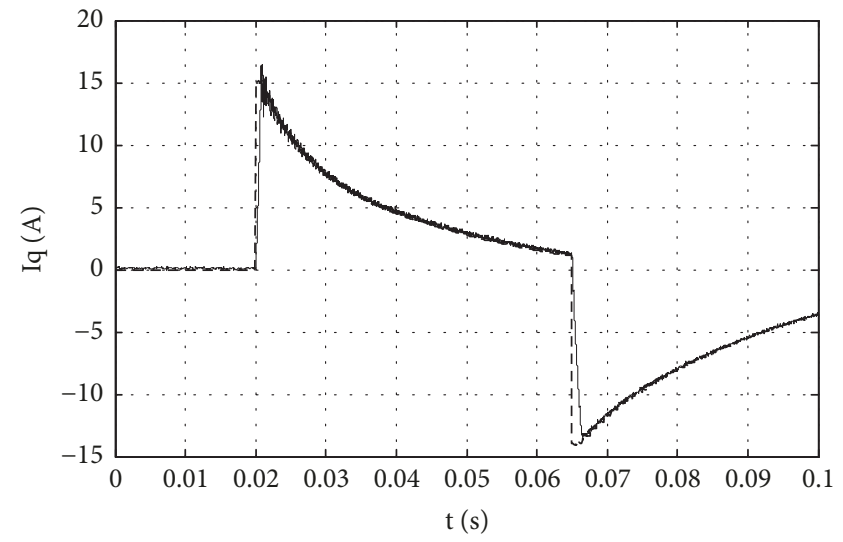

(a) $h=0.2$

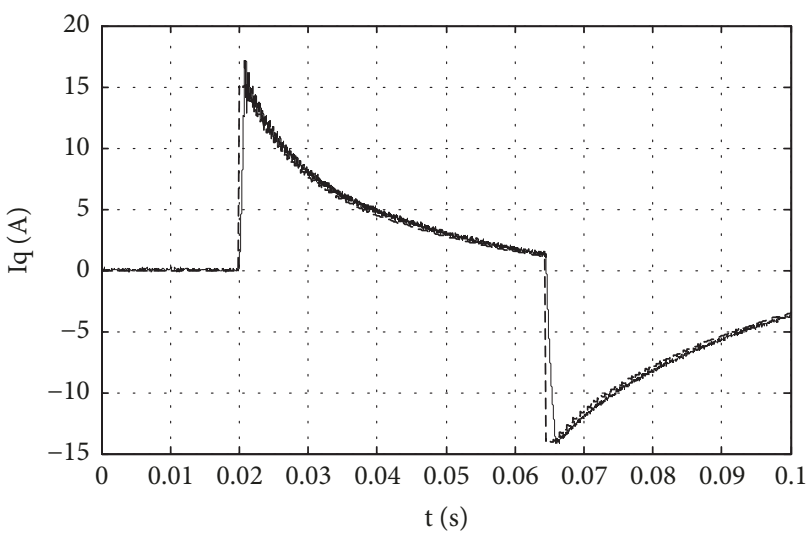

(b) $h=0.5$

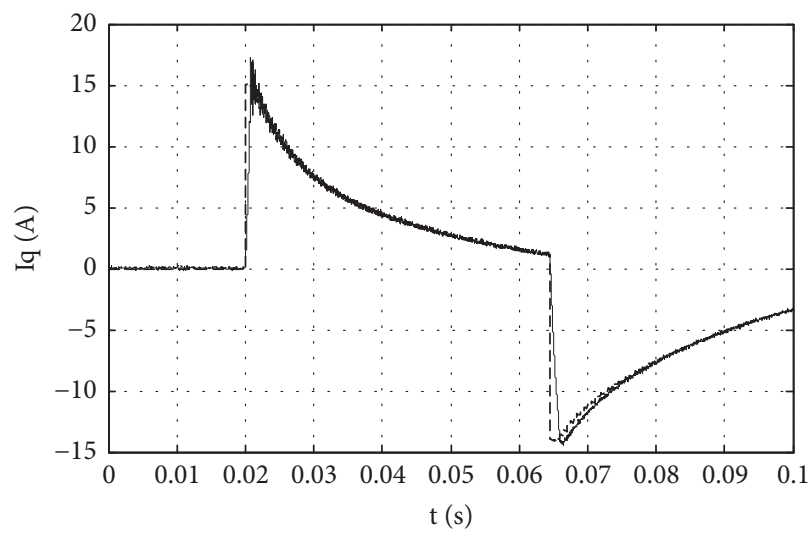

(c) $h=1.1$

FIGURE 12: Simulated results of quadrature axis current with sudden speed change at $0.02 \mathrm{~s}$ and $0.06 \mathrm{~s}$ for two-vector FCS-MPC based on duty ratio optimization with feedback correction, (a) $h=0.2$, (b), $h=0.5$, and (c) $h=1.1$. 


\section{Conflicts of Interest}

The authors declare that there are no conflicts of interest regarding the publication of this paper.

\section{Acknowledgments}

This work is supported by Research Foundation of China University of Petroleum-Beijing at Karamay (no. RCYJ2018A02-001).

\section{References}

[1] D.-C. Lee, S.-K. Sul, and M.-H. Park, "High performance current regulator for a field-oriented controlled induction motor drive," IEEE Transactions on Industry Applications, vol. 30, no. 5, pp. 1247-1257, 1994.

[2] P. Pillay and R. Krishnan, "Modeling, simulation, and analysis of permanent-magnet motor drives, Part I: The permanentmagnet synchronous motor drive," IEEE Transactions on Industry Applications, vol. 25, no. 2, pp. 265-273, 1989.

[3] K.-S. Low, K.-Y. Chiun, and K.-V. Ling, "Evaluating generalized predictive control for a brushless DC drive," IEEE Transactions on Power Electronics, vol. 13, no. 6, pp. 1191-1198, 1998.

[4] Y. Zhang and Q. Zhu, "Predictive function control based on the LS-SVM for marine steam turbine system," in Proceedings of the International Conference on Natural Computation, pp. 35083512, IEEE, Yantai, China, August 2010.

[5] K. Low, K. Chiun, and K. Ling, "A DSP-based servo system using generalized predictive control," in Proceedings of the Power Conversion Conference - PCC '97, pp. 507-512, IEEE, Nagaoka, Japan, August 1997.

[6] J. Zhang, X. Feng, S. Xiong, and Q. Wu, "Predictive function optimization control strategy of hydraulic servo system," in Proceedings of the 2014 33rd Chinese Control Conference (CCC), pp. 7758-7762, Nanjing, China, July 2014.

[7] Y. A.-R. I. Mohamed and E. F. El-Saadany, "Robust high bandwidth discrete-time predictive current control with predictive internal model - A unified approach for voltage-source PWM converters," IEEE Transactions on Power Electronics, vol. 23, no. 1, pp. 126-136, 2008.

[8] G. Wang, M. Yang, L. Niu, X. Gui, and D. Xu, "Static error elimination algorithm for PMSM predictive current control," in Proceedings of the 2014 33rd Chinese Control Conference (CCC), pp. 7691-7696, Nanjing, China, 2015.

[9] P. Wipasuramonton, Z. Q. Zhu, and D. Howe, "Predictive current control with current error correction for PM brushless AC drives," in Proceedings of the 2005 IEEE International Conference on Electric Machines and Drives, pp. 558-564, San Antonio, TX, USA, May 2005.

[10] Y. A.-R. I. Mohamed and E. F. El-Saadany, "An improved deadbeat current control scheme with a novel adaptive selftuning load model for a three-phase PWM voltage-source inverter," IEEE Transactions on Industrial Electronics, vol. 54, no. 2, pp. 747-759, 2007.

[11] J. Rodriguez and P. Cortes, "Predictive control of permanent magnet synchronous motors," in Predictive Control of Power Converters and Electrical Drives, pp. 133-144, John Wiley \& Sons, Ltd, Chichester, UK, 2012.

[12] R. N. Fard, Finite Control Set Model Predictive Control in Power Converters [Master thesis], Norwegian University of Science and Technology, 2013.
[13] M. Narimani, B. Wu, V. Yaramasu, C. Zhongyuan, and N. Reza Zargari, "Finite Control-Set Model Predictive Control (FCSMPC) of Nested Neutral Point-Clamped (NNPC) Converter," IEEE Transactions on Power Electronics, vol. 30, no. 12, pp. 72627269, 2015.

[14] S. Matsutani, T. Zanma, K. Kawai, M. Ishida, A. Imura, and M. Fujitsuna, "Optimal control of PMSMs using model predictive control," in Proceedings of 34th Annual Conference of IEEE on Industrial Electronics, pp. 1305-1310, Fukuoka, Japan, November 2008.

[15] X. Lin-Shi, F. Morel, A. M. Llor, B. Allard, and J.-M. Rétif, "Implementation of hybrid control for motor drives," IEEE Transactions on Industrial Electronics, vol. 54, no. 4, pp. 19461952, 2007.

[16] Y. Zhang and H. Yang, "An improved two-vectors-based model predictive torque control without weighting factors for induction motor drives," in Proceedings of the 17th International Conference on Electrical Machines and Systems, ICEMS 2014, pp. 2766-2772, Hangzhou, China, October 2014.

[17] Y. Zhang, W. Xie, Z. Li, and Y. Zhang, "Low-complexity model predictive power control: Double-vector-based approach," IEEE Transactions on Industrial Electronics, vol. 61, no. 11, pp. 58715880, 2014. 


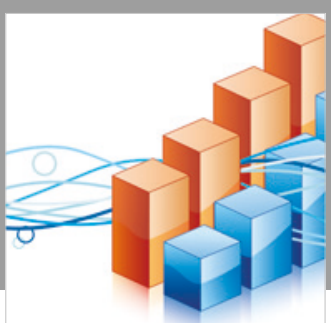

Advances in

Operations Research

\section{-n-m}
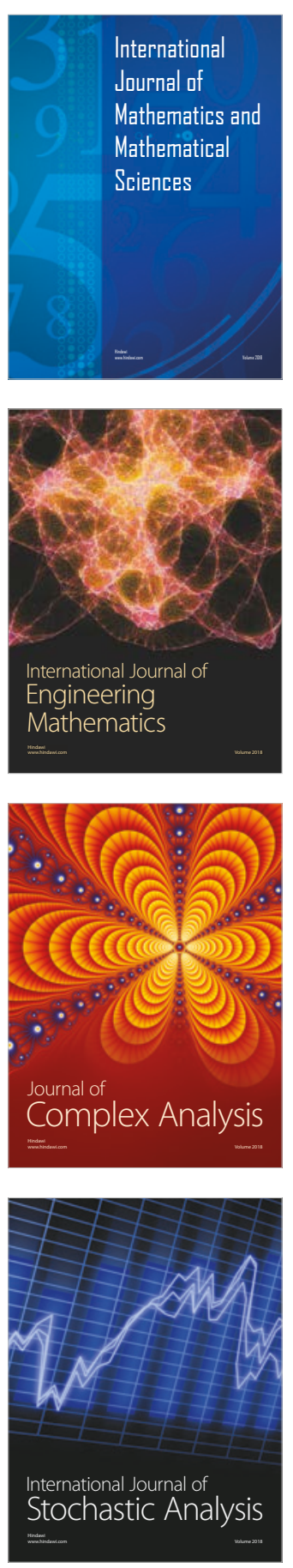
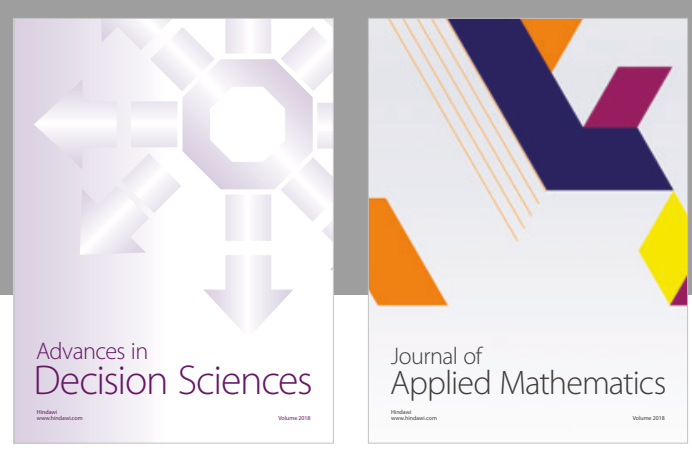

Journal of

Applied Mathematics
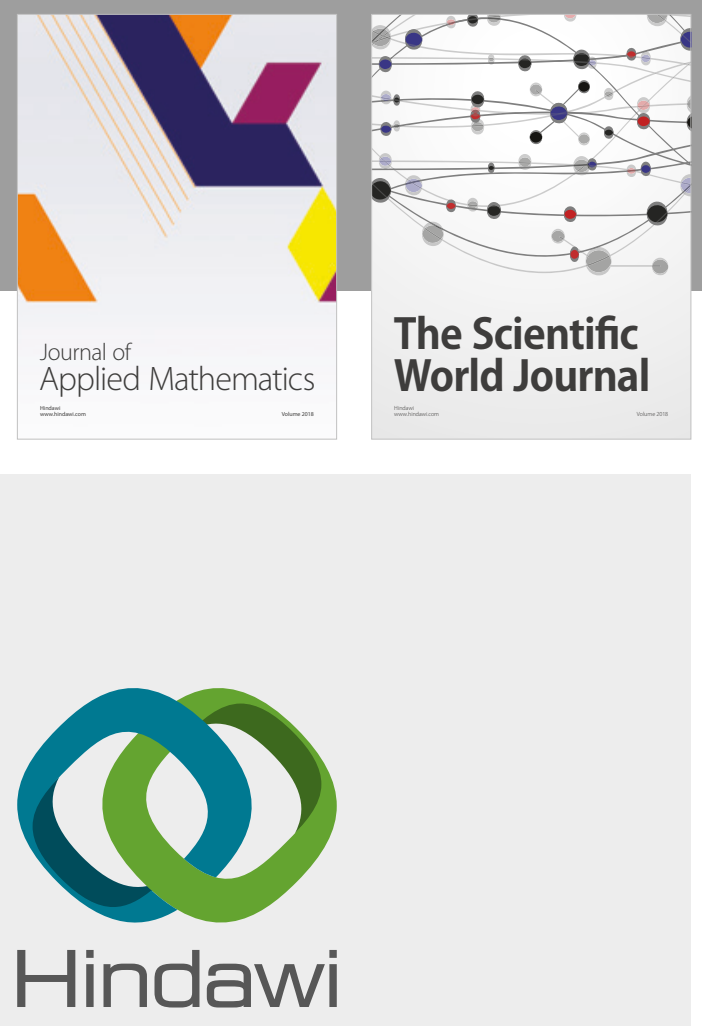

Submit your manuscripts at

www.hindawi.com

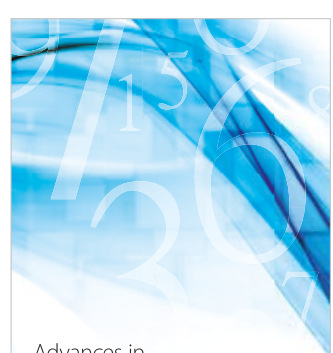

Advances in
Numerical Analysis
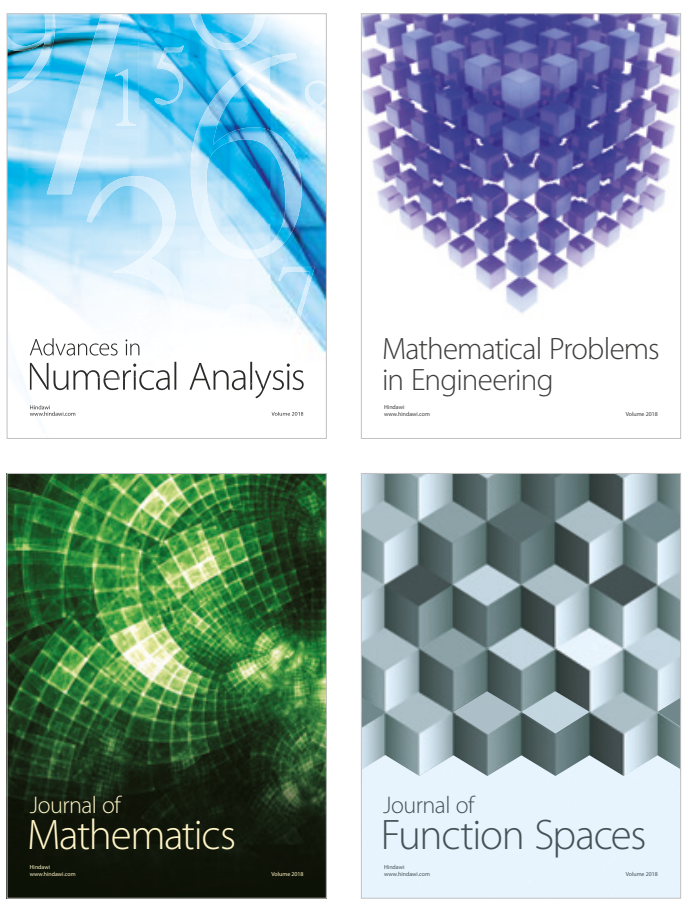

Mathematical Problems in Engineering

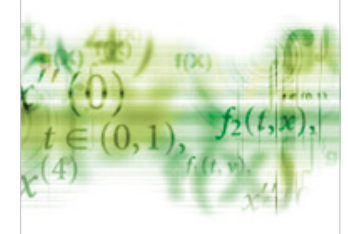

International Journal of

Differential Equations

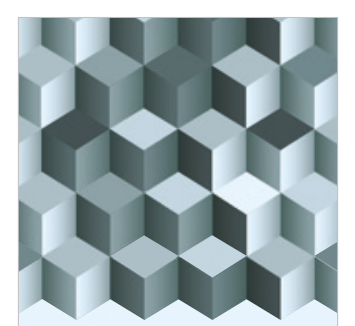

Journal of

Function Spaces

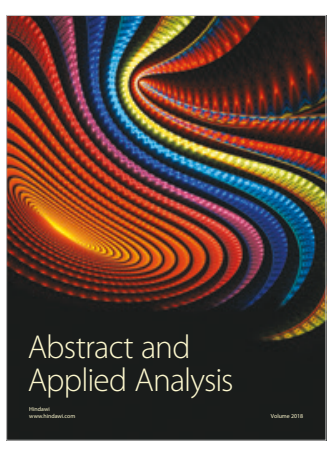

The Scientific

World Journal

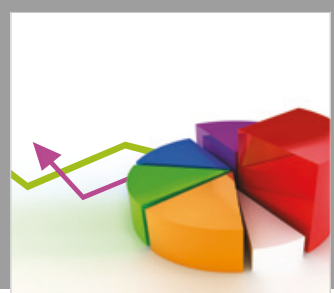

Journal of

Probability and Statistics
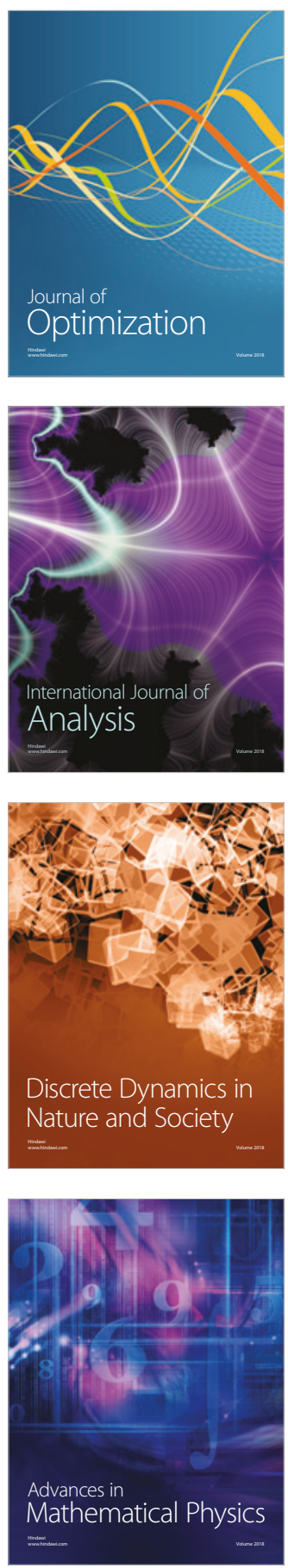